\title{
Dynamic Interaction Networks in Modelling and Predicting the Behaviour of Multiple Interactive Stock Markets
}

\author{
Harya Widiputra $^{1 *}$, Russel Pears ${ }^{1}$, Antoaneta Serguieva $^{2}$, Nikola Kasabov ${ }^{1}$ \\ ${ }^{1}$ Knowledge Engineering and Discovery Research Institute, Auckland University of \\ Technology, Private Bag 92006, Auckland 1020, New Zealand \\ ${ }^{2}$ Centre for Empirical Finance, Brunel University, and Brunel Business School, West \\ London, UB8 3PH, UK
}

\begin{abstract}
The behaviour of multiple stock markets can be described within the framework of complex dynamic systems (CDS). A representative technique of the framework is the dynamic interaction network (DIN), recently developed in the bioinformatics domain (Chan et al., 2006). DINs are capable of modelling dynamic interactions between genes and predicting their future expressions. In this paper, we adopt a DIN approach to extract and model interactions between stock markets. The network is further able to learn online and updates incrementally with the unfolding of the stock market timeseries. The approach is applied to a case study involving 10 market indexes in the Asia Pacific region. The results show that the DIN model reveals important and complex dynamic relationships between stock markets, demonstrating the ability of CDS approaches to go beyond the scope of traditional statistical methods.
\end{abstract}

Keywords: complex dynamic systems, interactive stock markets, dynamic interaction networks, online learning, time-series prediction.

\section{Introduction}

The globalised security markets of today are characterised with interdependences, and often demonstrate contagious behaviour in periods of crisis. An increasing number of studies are addressing the effect of such interrelationships, along with the challenge of relationship identification and modelling within a globalised environment. Chiang and Doong (2001) consider stock returns and volatility and find that four out of seven Asian markets present a significant relationship between stock returns and unexpected volatility. In their research on the French, German and UK indexes, and the corresponding stock index futures markets, Antoniou et al. (2003) signify that the behaviour of a domestic market is influenced by the foreign markets. Collins and Biekpe (2003) further study contagion and interdependence in African markets, finding evidence for contagion from the most traded markets. Serguieva and Wu (2007) and Caporale et al. (2008) focus on the Asian crisis of 1997. They use an agent-based approach to simulate contagion occurring during the crisis, and suggest a multinational mixed-game model to simulate the effect of multiple foreign markets on a domestic market.

\footnotetext{
*Corresponding author: email address: wpj6371@aut.ac.nz.
} 
Existing studies do not report the ability to simultaneously capture multiple dynamic relationships between interactive markets. This task serves as a motivation for our work, and we look at recent developments in the bioinformatics domain to relate the problem. Kasabov et al. (2004) and Kasabov (2007b) develop an approach based on Genetic Algorithms (Goldberg,1989) and the Kalman filter (Brown,1983) to identify interdependencies between genes. They use timeseries containing gene expression data collected over time intervals, and construct a gene regulatory network. If we associate gene expression values with stock market indexes, then the approach presents the potential to address the outlined problem. Furthermore, to meet the challenge of modelling and predicting multiple markets, it is essential to capture their interactive behaviour in a dynamic fashion. We propose a dynamic interaction network that analyses a set of timeseries data in the form of stock indexes, and reveals important interrelationships between them.

The study is focused on markets in the Asia Pacific region, and particularly analyses the stock indexes of Hong Kong, New Zealand, Australia, Japan, Indonesia, Malaysia, Singapore, Korea, Taiwan, and China. In section 2, we briefly describe the DIN extraction method and discuss how the dynamic interaction network can be used for prediction. Section 3 presents the results form the DIN experiments and their analysis. Finally, conclusions and directions for future research are outlined in section 4.

\section{Dynamic Interaction Network}

This section describes the basic building blocks in developing a dynamic interaction network. The Kalman filter is used as the mechanism for capturing interrelationships between markets.

\subsection{Modelling of DIN}

Existing research in bioinformatics (Chan et al.,2006; D'haeseleer et al.,1999) outlines the steps in extracting gene regulatory networks from time-course gene expression data, and shows that genetic network inference can be used successfully to reverse engineer the underlying regulatory interactions from the gene expression data. Developing the method further in this study, we extract dynamic interaction networks from interrelated timeseries data representing closing stock index values in markets form the Asia Pacific region. The method is based on the Kalman filter and the extracted DIN model is expected to reveal dynamic interactions among the stock markets. That will be useful in detecting influences and evaluating the degree to which a market influences or is being influenced by other markets. Through capturing dynamic influences among markets, we will be able to predict the behaviour of multiple stock indexes. Figure 1 exemplifies such behaviour with the trajectories of the markets indexes over a 108-week period spanning from May 2004 to November 2007. The selected ten markets in the Asia Pacific region include Australia (AORD), China (SSE), Hong Kong (HSIX), Indonesia (JSX), Japan (N225), Malaysia (KLSE), New Zealand (NZ50), Singapore (STI), South Korea (KOSPI), and Taiwan (TSEC). 


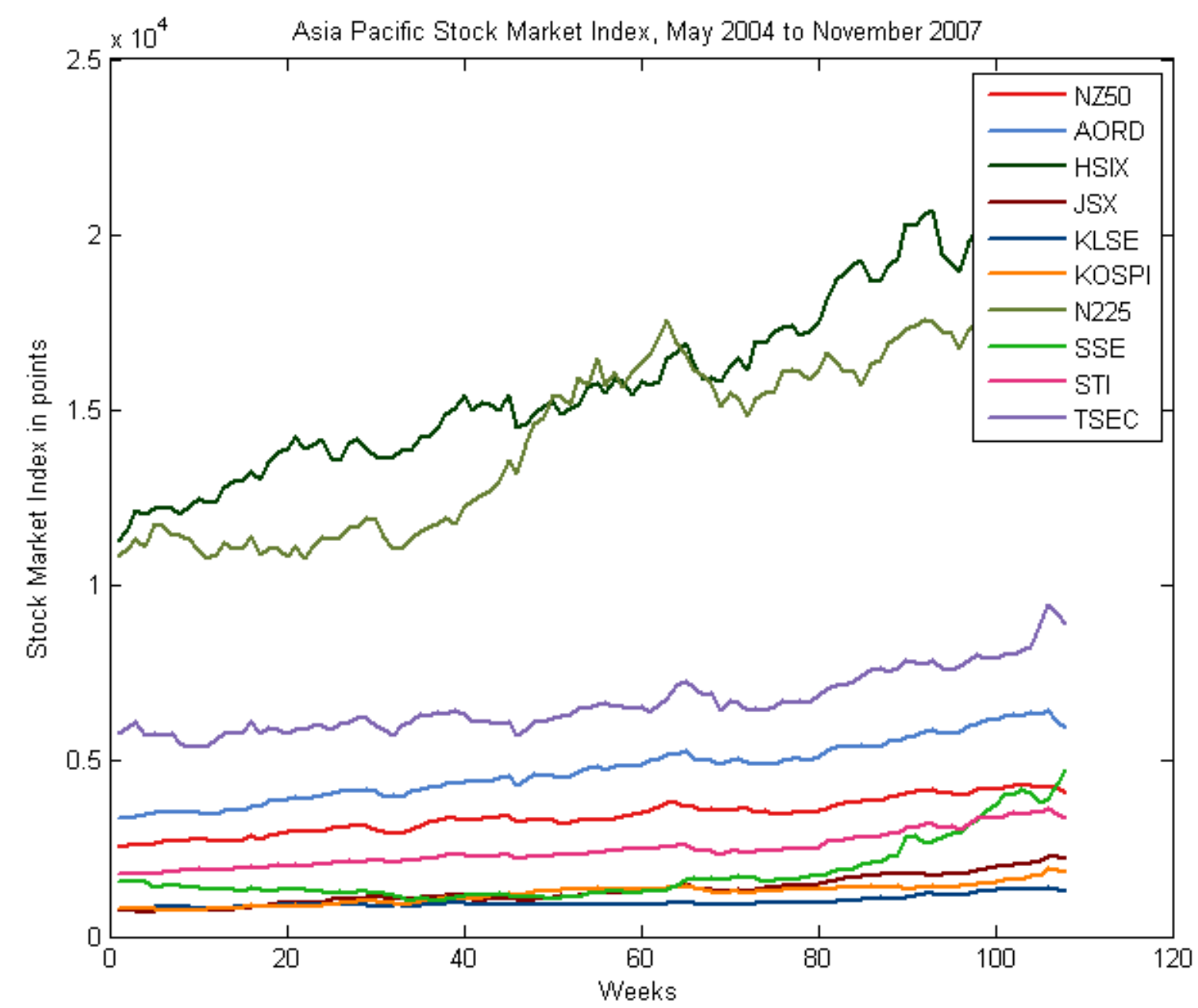

Figure 1: Stock market trajectories for markets in the Asia Pacific region

Discrete Kalman Filter Algorithm. The next step is introducing the algorithm based on the Kalman filter. The algorithm targets the general problem of estimating the state $x \in \Re^{n}$ of a discrete-time controlled process governed by the linear stochastic difference equation (1),

$$
x_{t}=A x_{t-1}+B u_{t-1}+w_{t-1},
$$

where $x$ is measured through $z \in \Re^{m}$ in equation (2),

$$
z_{t}=H x_{t}+v_{t} .
$$

The random variables $w_{t}$ and $v_{t}$ are assumed independent of each other, white, and with Gaussian probability distributions $p(w)$ and $p(v)$.

$$
\begin{aligned}
& p(w) \approx N(0, Q), \\
& p(v) \approx N(0, R) .
\end{aligned}
$$


The $n \times n$ matrix $A$ in equation (1) relates the state at time $t-1$ to the state at time $t$, in the absence of either a driving function or process noise. The Kalman filter (KF) estimates a process by using a form of feedback control (Welch and Bishop, 2006). The algorithm is divided in two parts: the time update equations and measurement update equations. The former can also be thought of as predictor equations, while the latter can be considered as corrector equations. Thus the overall algorithm resembles a predictor-corrector algorithm for solving numerical problems. The KF operation is summarised in Figure 2.

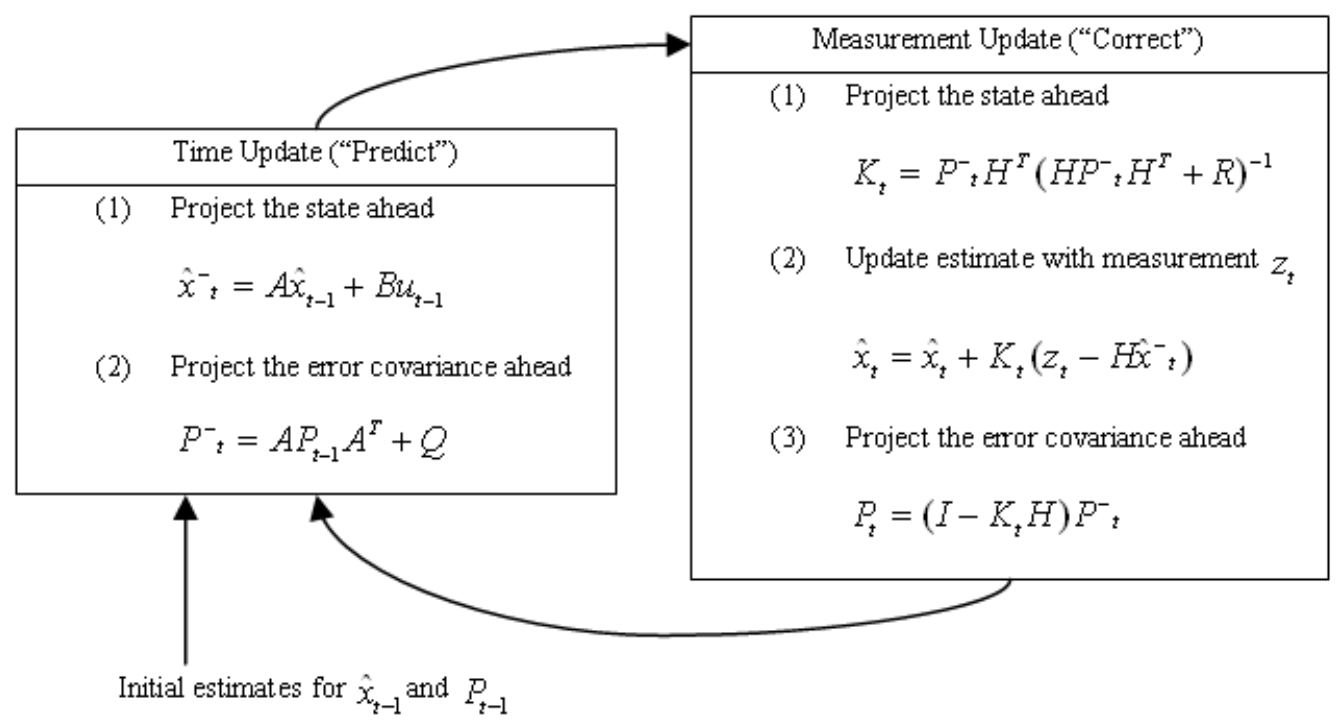

Figure 2: Operation of the KF algorithm

The $n \times n$ transition matrix $A$ is used to extract the DIN model from the analysed interrelated timeseries. Constructing a dynamic interaction network from a transition matrix is exemplified in Figure 3. We include three of the markets in the exemplary DIN: China, Taiwan, and Hong Kong.

(a)

$$
\left[\begin{array}{ccc}
0.53 & -0.23 & 0.36 \\
-0.01 & 0.87 & 0.43 \\
0.52 & -0.02 & 0.31
\end{array}\right]\left[\begin{array}{ccc}
+ & - & + \\
0 & + & + \\
+ & 0 & +
\end{array}\right]
$$

(b)

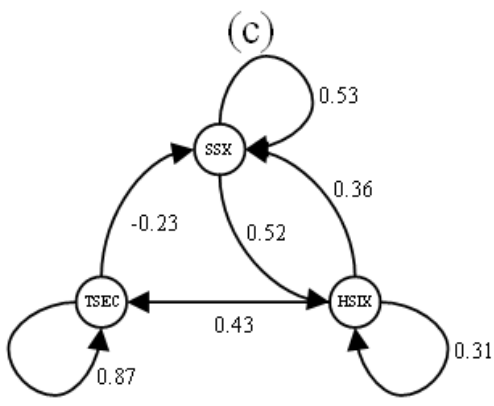

Figure 3: Constructing a DIN model

(a) the transition matrix $A$;

(b) the corresponding influence matrix;

(c) the network diagram. 
Stock market relationships are elucidated from the transition matrix using a threshold. All transitions $x_{i j}$ with values in $A$ greater than 0.1 are flagged as being positive, i.e. market $i$ is influencing market $j$. By analogy, values below -0.1 are labelled as negative. The values and direction of influence are reflected in the directed network diagram.

\subsection{Prediction with DIN}

We use two different approaches to prediction. First, the network extracted from the transition matrix is applied to predict future states of the system, while the matrix itself is not updated. Next, the transition matrix is updated with available new data. The second approach can be considered as prediction with online learning.

Offline Learning. Here, the transition matrix $A$ is the starting point to predict future movements in the stock market indexes, by relating the state at time step $t$ to the state at the future time step $t+1$. As shown in Figure 4, a model is induced from training data covering a period of 50 weeks, and the model is then used for prediction over the next 20 weeks.

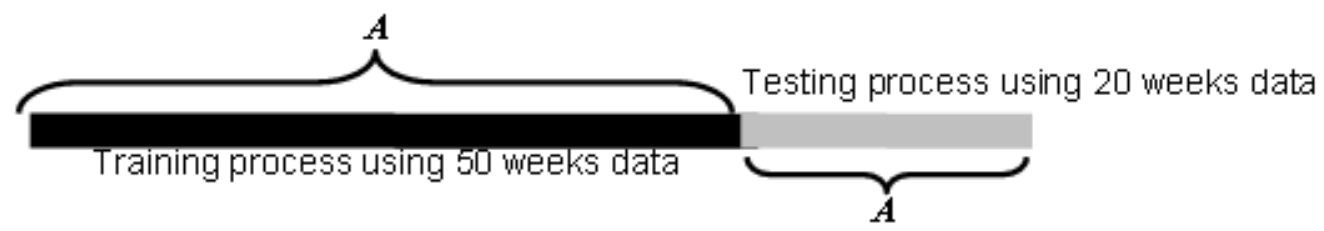

Figure 4: Prediction with offline learning

Online Learning. Systems that learn incrementally with incoming data and evolve their structure and functionality are referred to as evolving systems. An evolving system can work in online mode operating with real-time data, e.g. evolving connectionist systems (Kasabov, 2002 and 2007b). Correspondingly, in this study we implement a prediction process with online learning, where the transition matrix and the DIN model are updated at any time step. As shown in Figure 5, a model is again trained over a certain number of weeks, e.g. 50 weeks of data. This model is used to predict the next week's index values. Then, the model is updated next week with the new data arriving, and the updated model is used to predict index values for the week after. The incremental process continues into the future, and testing takes place together with training on new data.

Testing process using 20 weeks data

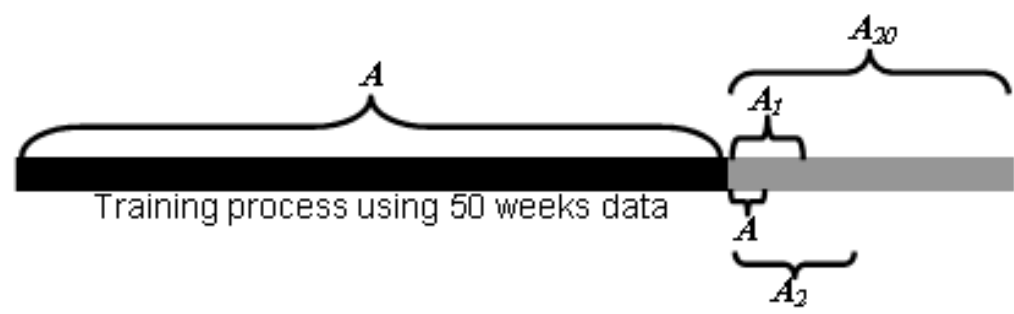

Figure 5: Prediction with online learning 


\section{Experiments and Results}

Our dataset includes index timeseries for ten stock markets in the Asia Pacific region ${ }^{1}$, spanning seventy weeks from June 2005 to June 2007. We use weekly aggregated values and normalize the data to lie in the range $[-1,1]$. The selected ten market indexes are AORD (Australia), HSIX (Hong Kong), JSX (Indonesia), KLSE (Malaysia), KOSPI (South Korea), N225 (Japan), NZ50 (New Zealand), SSE (China), STI (Singapore), and TSEC (Taiwan).

\subsection{Model Analysis}

To extract a reliable model, we run 10 different trials on the training dataset and average each of the values in the transition matrix before constructing the dynamic interaction network. The results of the modelling process are: (1) the transition matrix, (2) the DIN diagram of interactive stock markets, and (3) a graph comparing actual (solid-line) and simulated (dashed-line) index trajectories. We use the DIN Extraction Software (DRESt), and a result screen is presented in Figure 6.

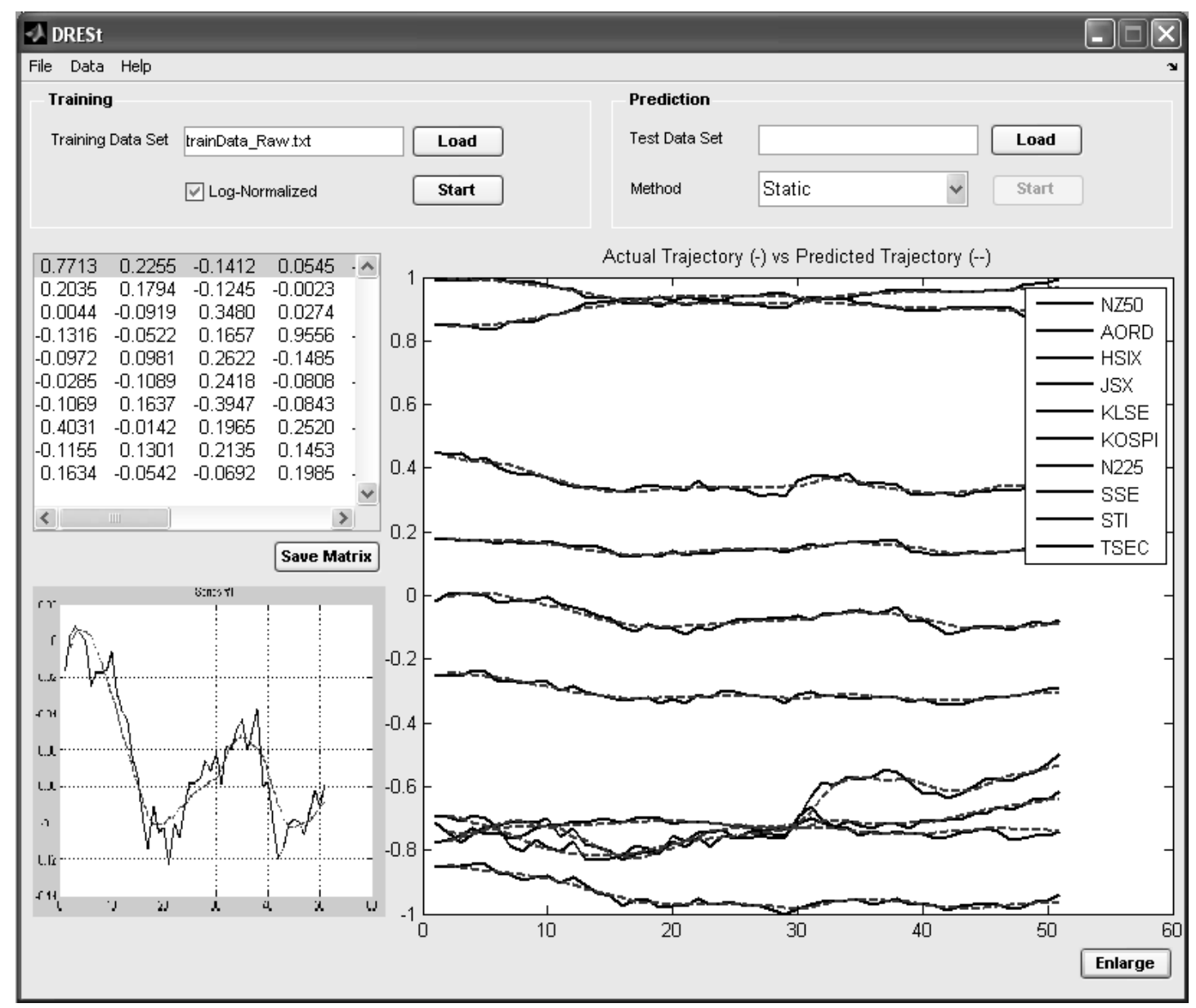

Figure 6: DRESt - the DIN Extraction Software

\footnotetext{
${ }^{1}$ Available from http://finance.yahoo.com/intlindices?e=asia.
} 
Figure 7 presents the results of the DIN modelling process. Part (c) of the figure clearly shows that the estimated trajectories, based on the extracted DIN, closely track the actual trajectories. Therefore, the first-order Kalman-filter difference equations are a good approximator for future values of multiple stock indexes.

(a)

$$
\left[\begin{array}{cccccccccc}
0.77 & 0.22 & 0 & 0 & 0 & 0 & 0 & 0 & 0 & 0 \\
0.2 & 0 & 0 & 0 & 0 & 0 & 0 & 0 & 0 & 0.24 \\
0 & 0 & 0.35 & 0 & 0 & 0 & -0.37 & 0 & 0 & 0 \\
0 & 0 & 0 & 0.96 & -0.27 & -0.24 & 0 & 0 & 0 & 0 \\
0 & 0 & 0.26 & 0 & 0.41 & 0 & 0 & 0 & 0.24 & 0.34 \\
0 & 0 & 0.24 & 0 & -0.24 & 0.69 & 0 & 0 & 0 & 0 \\
0 & 0 & -0.4 & 0 & 0 & 0 & 0.54 & 0 & 0 & 0 \\
0.42 & 0 & 0.21 & 0.27 & -0.8 & 0.59 & -0.22 & 0.81 & -0.32 & 0.49 \\
0 & 0 & 0.22 & 0 & 0 & 0 & 0 & 0 & 0.44 & 0 \\
0 & 0 & 0 & 0 & 0 & 0.21 & 0 & 0 & 0 & 0.89
\end{array}\right]
$$

(c)
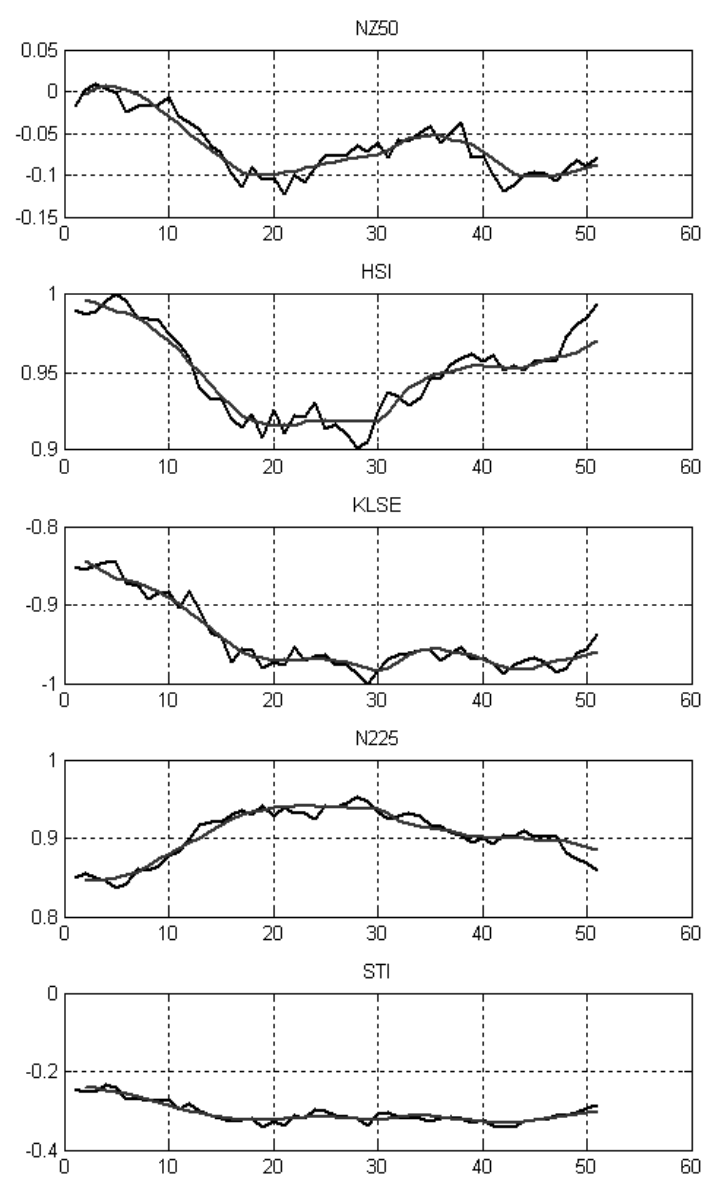

(b)
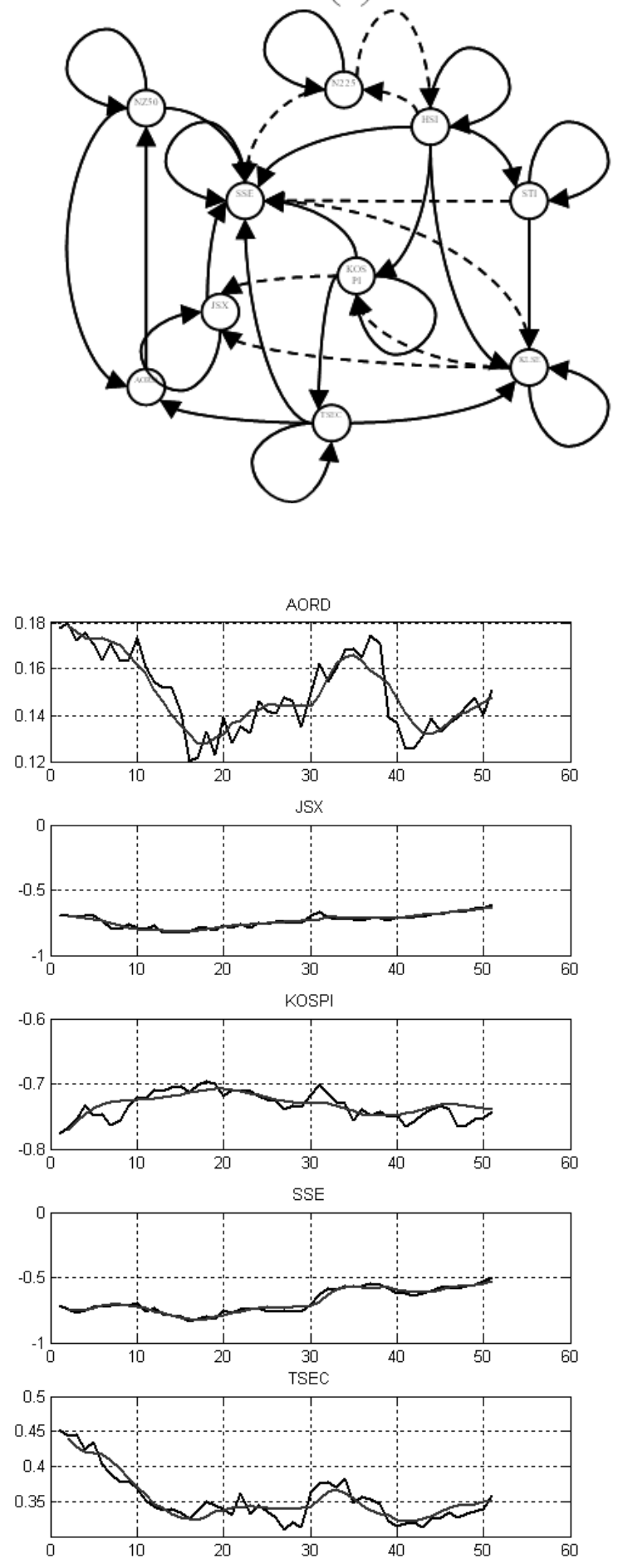

Figure 7: Results of the DIN modelling process

(a) transition matrix; (b) DIN diagram; (c) actual and KF-estimated trajectories. Note: Dashed edges in the interaction network diagram represent negative relationships between vertices. 
Furthermore, the DIN model reveals that Hong Kong (HSIX), Taiwan (TSEC), Singapore (STI), South Korea (KOSPI), and Japan (N225) are influential markets in the region. This is in agreement with those markets being based in the economic powerhouses of the Asia Pacific region and tending to influence countries economically related to them. For instance, Figure $7 \mathrm{~b}$ recognises the influence that Hong Kong exerts on China (Shanghai stock market), Japan, Korea, Malaysia, and Singapore.

Secondly, the model shows that China (SSE) is affected by most of the other markets. To analyse the result, we need to consider China's strategic approach to its Asian neighbours. China: (1) has become one of the largest traders and investors with many Asian countries; (2) exports primarily consumer goods to most countries in Asia Pacific; (3) is more than just a trading partner but also extensively invests in the region (e.g. China recently became one of the largest investors in Indonesia buying into oil and gas interests); and (4) is the largest foreign investor in some of the smaller economies in Southeast Asia. By proposing to negotiate a free trade agreement with the ASEAN countries, China offered to share the benefits of its economic growth, while reminding the region of their growing reliance on China. All these contribute to the unique position of China, and to the number of vertices involved in the diagram.

Next, we find that the network model also identifies interactions between Taiwan (TSEC), Hong Kong (HSIX), Singapore (STI), South Korea (KOSPI), and Australia (AORD), which is in agreement with previous findings by Masih and Masih (1998) in their research on the dynamics of stock market interdependency. Though their results are based on the period from 1982 to 1994, we conclude that the relationships among these countries are preserved in the more recent period. Therefore, for some leading economies with relatively stable economic infrastructure, consistent interactions exist in terms of stock market relationships.

Considering the level of integration of the economies in the region, particularly China and Southeast Asia, we recognise that as a factor for the extracted interrelations between the financial markets. Another contributing factor is their significant dependence on the US economy and market, which can be considered as a common outside player. In this paper, we focus on modelling the behaviour of Asia Pacific markets as an interactive regional system, being aware of the different contributing causes. At a future point, we will explicitly introduce the US market into the model to observe and analyse the redistribution of influences.

In conclusion, the transition matrix in Figure 7a represents meaningful relationships between the variables in the training dataset, and can be used to build a DIN model as shown in Figure 7b. We also analyse how the DIN model changes when new data become available. An experiment is run where the DIN model is extracted after 10 weeks, and then again after further 10 weeks. The results are shown in Figure 8. Feeding 10 weeks of new data leads to significant changes in the DIN. The most significant change is the disappearance of interaction between New Zealand (NZ50) and Australia (AORD). A closer look at the trajectories reveals that for the 10 weeks of new data, NZ50 and AORD move quite independently of each other, in contrast to their behaviour in the previous 10 weeks. Another noteworthy change in Figure $8 \mathrm{~b}$ is that after feeding 20 weeks of new data, the interaction between NZ50 and AORD 
reappears. Figure 10 is relevant here, as it shows that starting from week 12 , upward movement in the NZ50 index is accompanied by corresponding increases in the AORD index and vice versa, restoring the linkage between the two markets. Therefore, the DIN model adapts to changes in stock market conditions, and faithfully represents the interactions among markets.

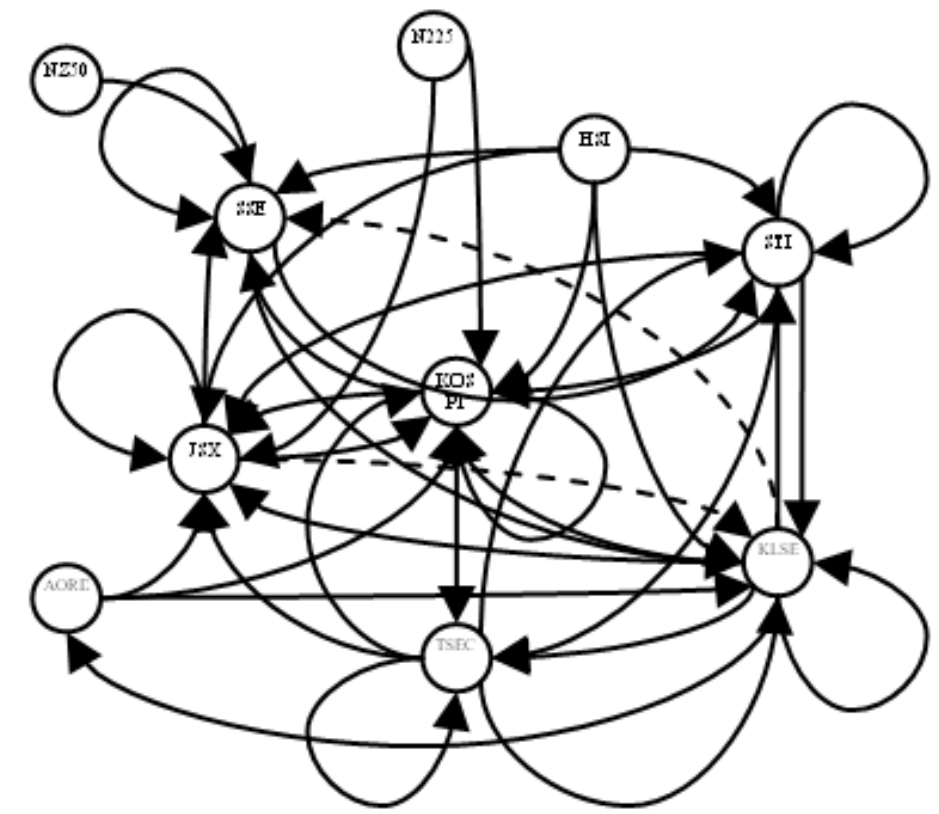

(b)

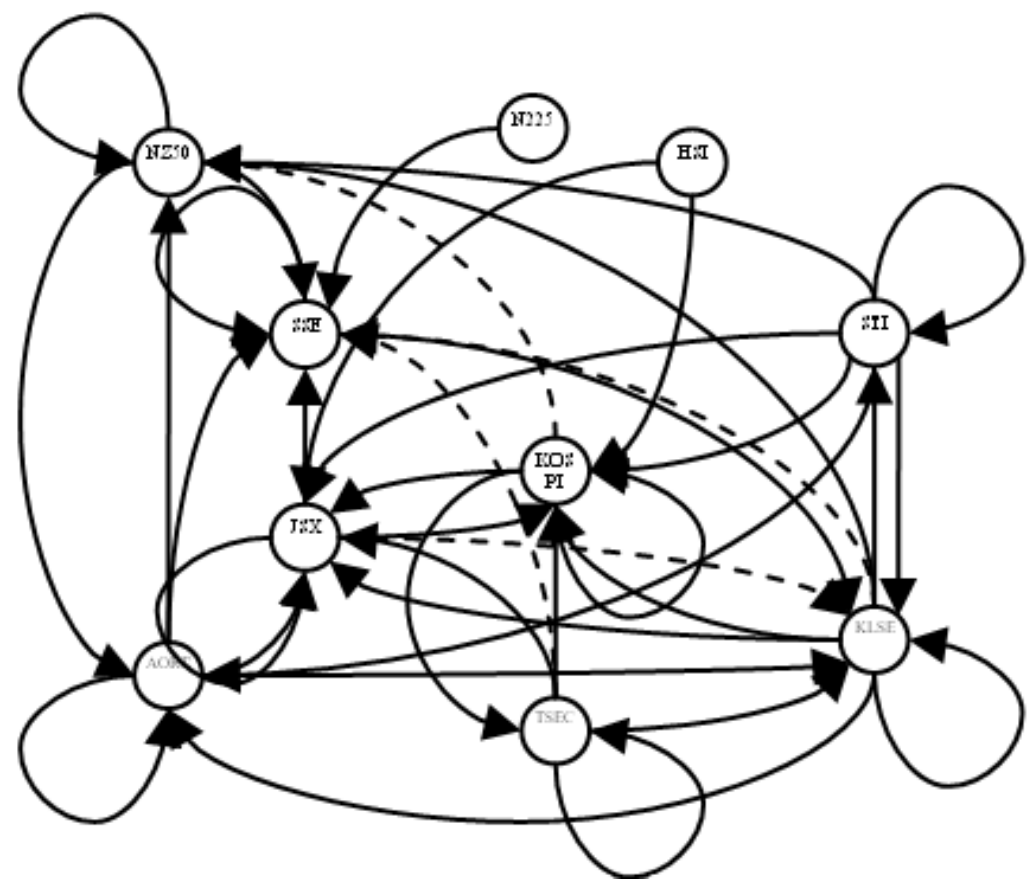

Figure 8: Feeding new data and updating the DIN model

(a) DIN model after 10 weeks;

(b) DIN model after 20 weeks.

Note: Dashed edges show inverse relationships between vertices. 
For comparison, we fit in equation (5) a multiple linear regression model for the NZ50 index. The model shows that NZ50 is dependent on AORD, KLSE and SSE. This is broadly consistent with the extracted DIN. However, the DIN model has a much better goodness of fit than the regression, as shown in Figure 9.

$$
N Z 50=0.674 A O R D+0.346 K L S E-0.126 S S E
$$

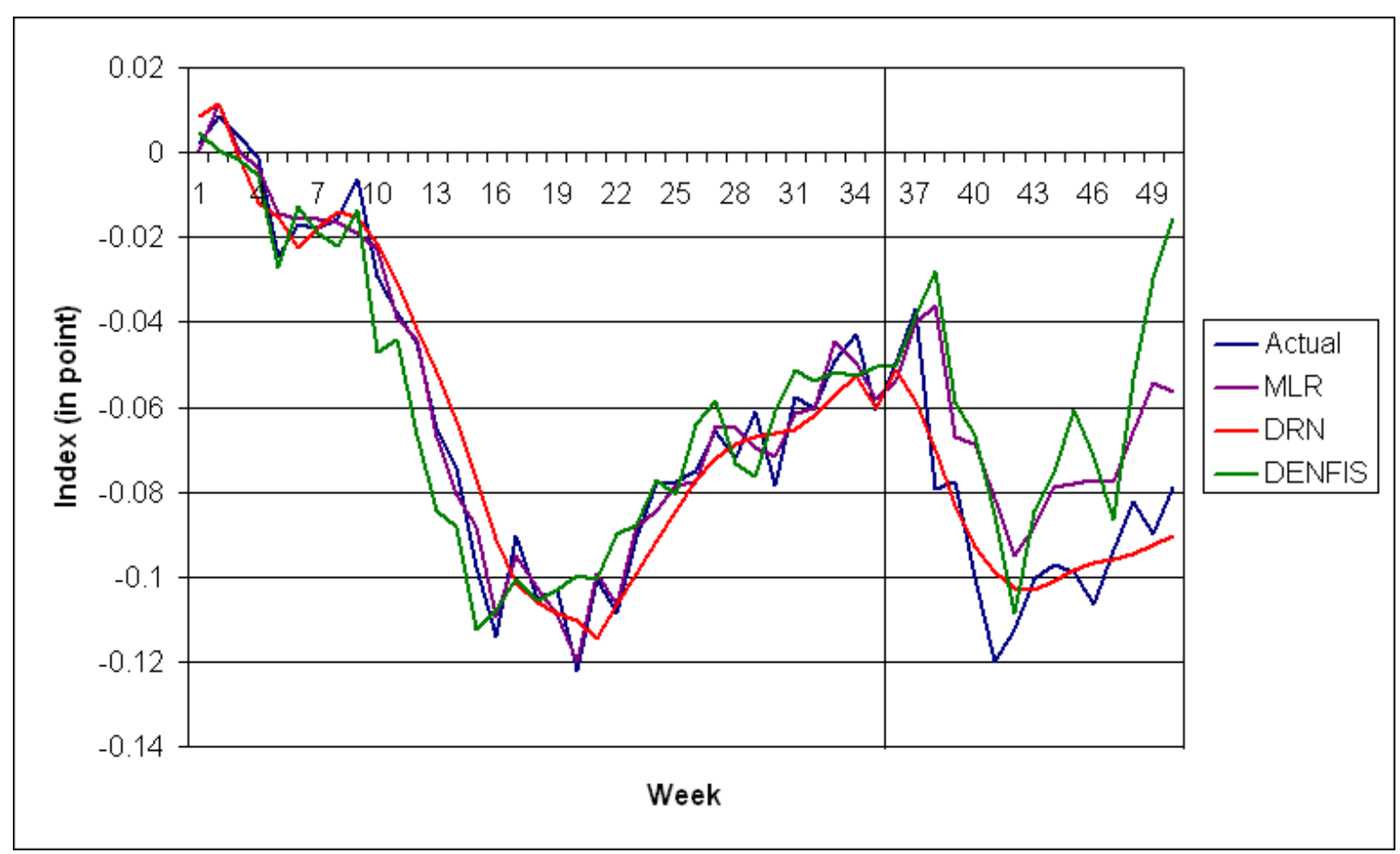

Figure 9: Actual NZ50 versus Predicted NZ50 with Multiple Regression, DIN, and DENFIS [18]

Furthermore, we calculate the correlation coefficients between each two markets (see Table 1) using the adjusted correlation that accounts for differences in heteroscedasticity (Collins and Biekpe, 2003). For example, we recognise that NZ50 is related to AORD with a coefficient of 0.94 and to HSIX with 0.67 , while AORD is related to HSIX with a coefficient of 0.65 and to JSX with 0.20 .

Table 1: Correlation Coefficient Matrix

\begin{tabular}{|c|c|c|c|c|c|c|c|c|c|c|}
\hline & IZ5 & $A O R$ & 75 & $7 S X$ & $K L$ & $K C$ & $P I N 22$ & SSE & $S T I$ & TSEC \\
\hline 50 & 1.00 & 0.94 & 0.67 & -0.05 & 0.90 & -0.33 & -0.71 & -0.19 & 0.87 & $0.86^{\circ}$ \\
\hline & 0.94 & 1.00 & 0.65 & 0.20 & 0.77 & -0.39 & -0.64 & 0.24 & 0.79 & 0.84 \\
\hline$H S L X$ & 0.67 & 0.65 & 1.00 & 0.58 & 0.76 & -0.72 & -0.99 & 0.55 & 0.76 & 0.68 \\
\hline$J S X$ & -0.05 & 0.20 & 0.58 & 1.00 & -0.11 & -0.64 & -0.43 & 0.89 & 0.15 & 0.17 \\
\hline$K L S E$ & 0.90 & 0.77 & 0.76 & -0.11 & 1.00 & -0.29 & -0.84 & -0.40 & 0.94 & 0.89 \\
\hline KOSPI & -0.33 & -039 & -0.72 & -0.64 & -0.29 & 1.00 & 0.70 & -0.68 & -0.34 & -0.31 \\
\hline$N 225$ & -0.71 & -0.64 & -0.99 & -0.43 & -0.84 & 0.70 & 1.00 & -0.34 & -0.81 & -0.74 \\
\hline$S S E$ & -0.19 & 0.24 & 0.55 & 0.89 & -0.40 & -0.68 & -0.34 & 1.00 & -0.20 & -0.17 \\
\hline$S T I$ & 0.87 & 0.79 & 0.76 & 0.15 & .94 & -0.34 & -0.81 & -0.20 & 1.00 & 0.91 \\
\hline$T S E C$ & 0.86 & 0.84 & 0.68 & 0.17 & 0.89 & -0.31 & -0.74 & -0.17 & 0.91 & 1.00 \\
\hline
\end{tabular}


However, we cannot infer that NZ50 is related in that way to both AORD and HSIX, and at the same time AORD is related in that way to both HSIX and JSX. Alternatively, the proposed method here differs from correlation analysis, and reveals relationships that exist between multiple interactive variables across time.

\subsection{Prediction Results}

We test the extracted DIN model to predict future values of multiple stock indexes. The test dataset covers twenty weeks, following chronologically the 50 weeks of training data. First, we consider the goodness of fit of the DIN model at all 20 points of the test dataset, plotting actual test trajectories and simulated trajectories. Second, we calculate the prediction error, using the root mean squared error, to measure how good the DIN prediction performance is. The plot of actual and estimated trajectories in Figure 10 shows only slight differences, and therefore satisfactory prediction results. This is confirmed by the error rates presented in Table 2. We can conclude that using the previous fifty weeks as a training dataset, we have extracted a reliable DIN model. The model represents relationships among stock markets which are sufficiently accurate to be used for predicting future values.

Table 2: Error rates for offline prediction

\begin{tabular}{|c|c|c|c|c|c|c|c|c|c|}
\hline \multicolumn{1}{|c|}{ RMSE } \\
\hline N750 & AORD & HSX & JSX & KLSE & KOSPI & N225 & SSE & STI & TSEC \\
\hline 0.0212 & 0.0296 & 0.0113 & 0.0430 & 0.0486 & 0.0547 & 0.0134 & 0.0767 & 0.0392 & 0.0462 \\
\hline
\end{tabular}

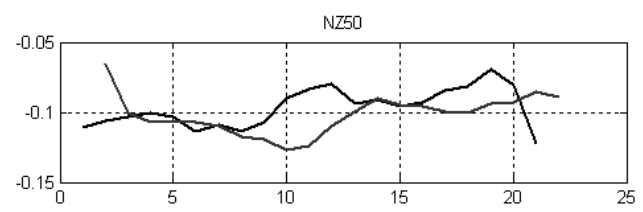

$\mathrm{HSI}$

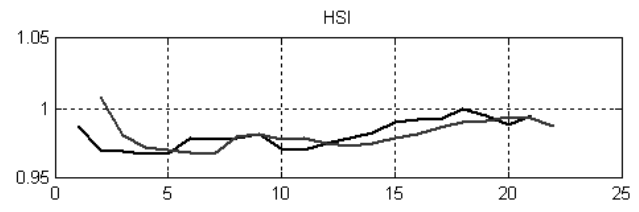

KLSE

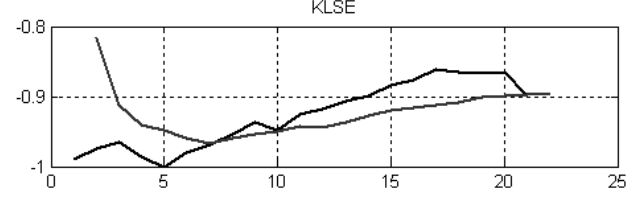

N225

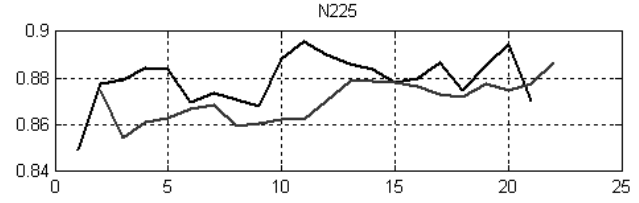

STI

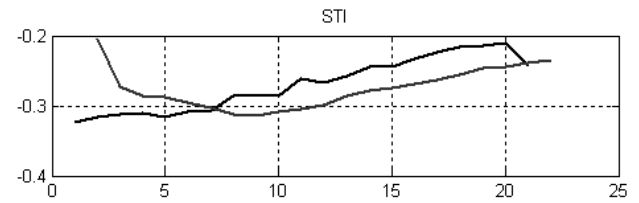

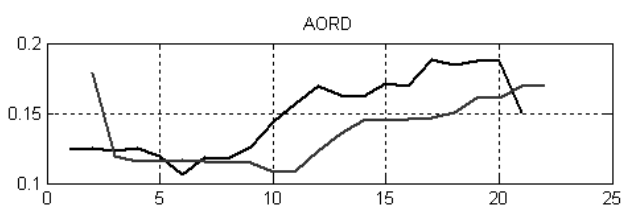

JSX

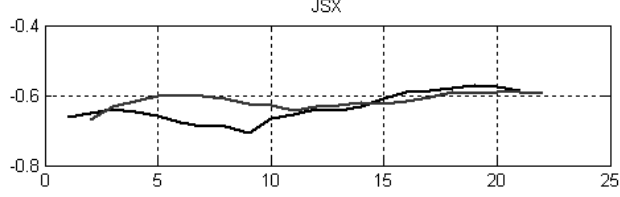

KOSPI

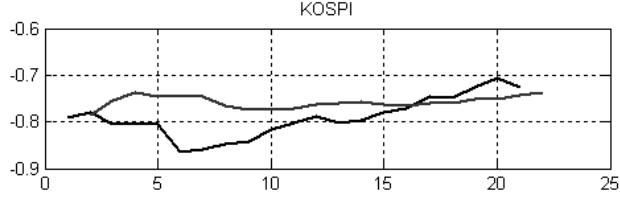

SSE

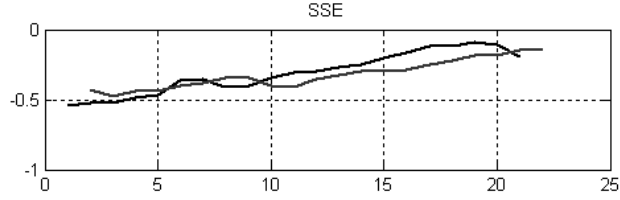

TSEC

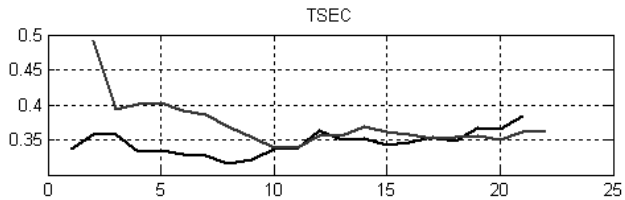

Figure 10: Comparison between actual (-) and predicted (- -) trajectories over the 20-week test dataset 
Next, we investigate the possibility of gaining new knowledge on market interrelationships as market conditions change. The knowledge is updated incrementally through online predictive learning. The trajectories generated with the online DIN model are presented in Figure 11, showing that the dynamic interaction network extracted through online learning achieves better accuracy of prediction. The result is confirmed by the error measurements in Table 3, which are significantly lower in comparison with the error rates in Table 2.

Table 3: Error rates for prediction with online learning

\begin{tabular}{|c|c|c|c|c|c|c|c|c|c|}
\hline \multicolumn{10}{|c|}{ RMSE } \\
\hline NZ50 & AORD & HSIX & JSX & KLSE & KOSPI & N225 & SSE & STI & TSEC \\
\hline 0.0117 & 0.0108 & 0.0066 & 0.0123 & 0.0143 & 0.0140 & 0.0076 & 0.0375 & 0.0097 & 0.0142 \\
\hline
\end{tabular}

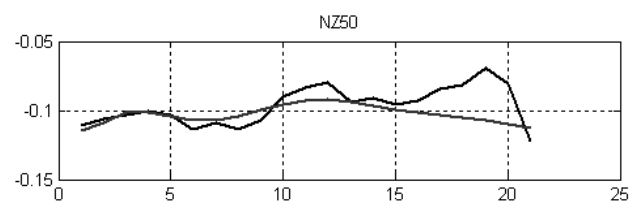

$\mathrm{HSI}$
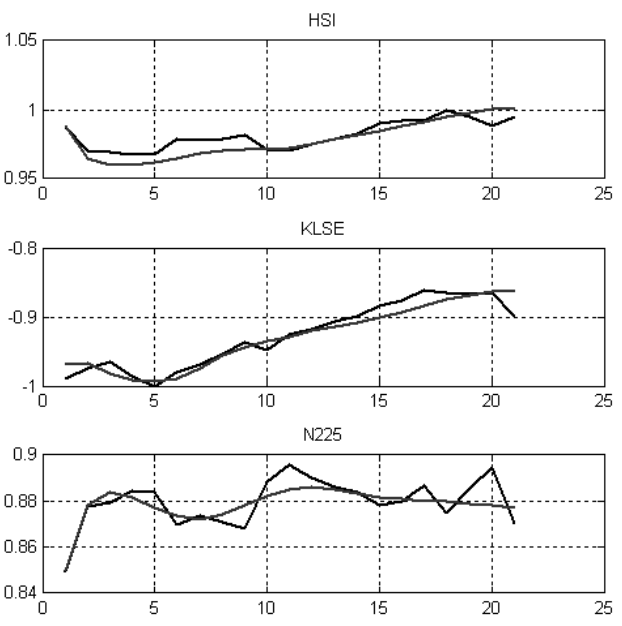

STI

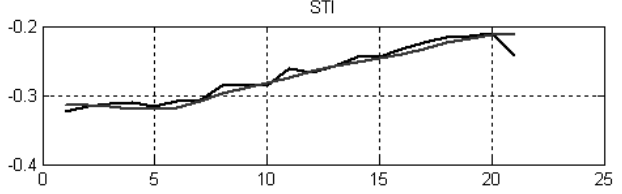

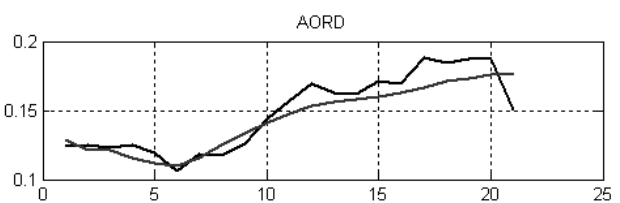

JSX

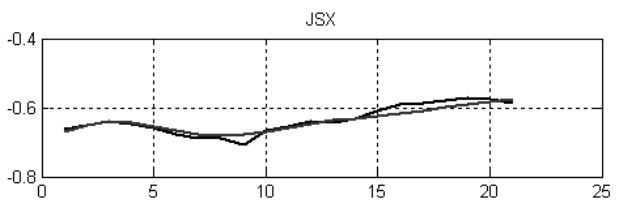

KOSPI

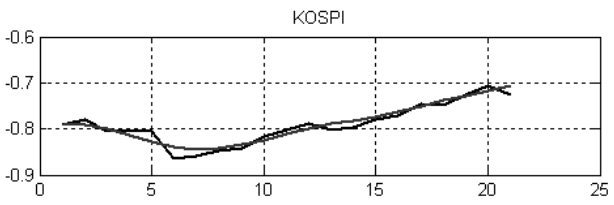

SSE

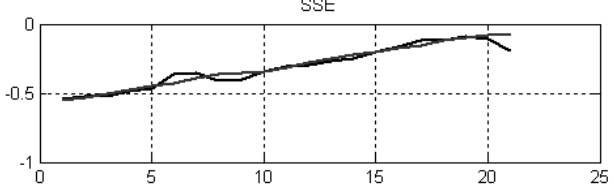

TSEC

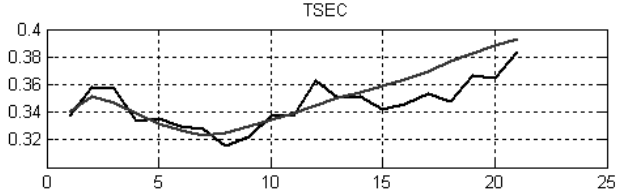

Figure 11: Comparison between actual (-) and predicted (--) trajectories with online learning over the 20-week test dataset

\subsection{Long-term Relationship Analysis using a Markov Chain}

The DIN model analysis in section 3.1 has revealed that the relationships between markets changes through time, as depicted in Figure $8 \mathrm{a}$ and Figure 8b. In view of determining the long-term relationships between markets, we introduce a Markov Chain process (Hamilton, 2005) and use a longer dataset. For each of the 10 stock markets, 108 weekly data are stored in a separate dataset, giving rise to 108 datasets each comprising the 10 index values. After bootstrapping a DIN model trained on the first 10 weeks of data, we use a sliding window of size 10 to keep the 10 most recent weeks of data for training. After extracting the 108 instances of DIN, we plot the coefficients in the model. For example, Figure 12 shows how the 
relationships between New Zealand's market (NZ50) and other markets (including itself) changes over time. The straight line in each graphics indicates a threshold calculated as the mean value of a coefficient. This then serves as a reference point, and a relationship can be considered strong if the corresponding coefficient value is above the threshold, while it is considered weak if below the threshold.

For example, the relationship between NZ50 and AORD (Australia) stays strong for longer in some periods (weeks 21-23, 64-71, and 104-108); and keeps changing between strong and week in the rest of the time. Analogical character is displayed by the other coefficients, each staying strong for longer in different periods, and then keep changing between strong and week. Therefore, the behaviour of NZ50 will be better described if considering its relationships with all indexes rather than a single index. Still, the results in Figure 12 reveal that the relationships between markets change dynamically over time without particularly identifiable patterns. It presents a challenge for a single (though adaptive) global model to accurately predict movements of all stock indexes at all points in time. The accuracy could be improved further if a multiperspective modelling process is adopted using problem subspaces of varying size (Serguieva et al.,2003; Serguieva and Khan,2004) where our global model is augmented with local and personalised models (Kasabov, 2007a and 2007b) adapting better to changing market conditions. This presents a direction for our future research.
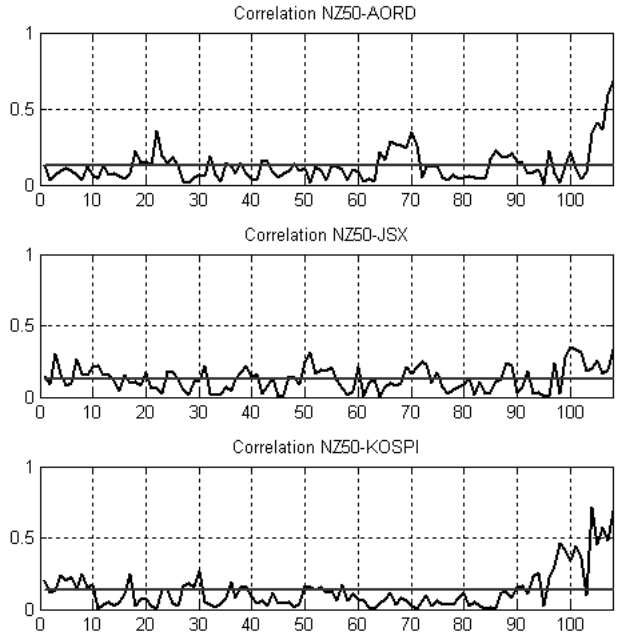

Correlation NZ50-NZ50

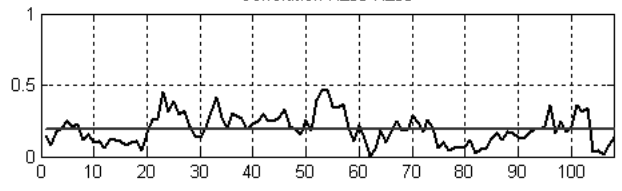

Correlation NZ50-STI

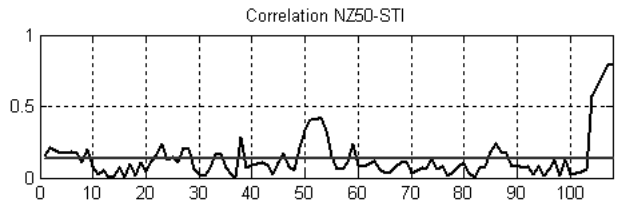

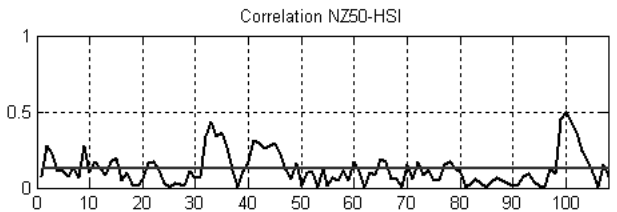

Correlation NZ50-KLSE

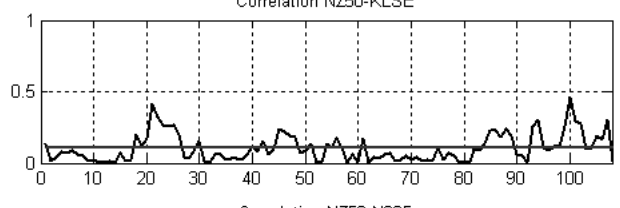

Correlation NZ50-N225

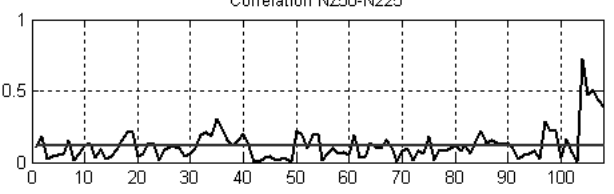

Correlation NZ50-SSE
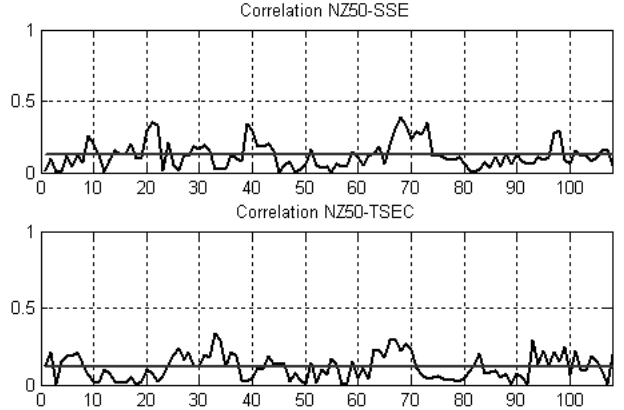

Figure 12: NZ50 coefficients from 108 dynamic interaction networks

Next, we use thresholding on the coefficient in the KF transition matrix, in order to estimate the probability of markets being in a long-term relationship with each other. Each coefficient value is mapped to a new value using Rule 1, and Figure 13 shows the result of applying the Markov Chain model with the thresholding rule. We 
consider the NZ50 index again and calculate, over the next 50 weeks, the probabilities of relationships existing with other markets. This is based on the behaviour of relationship coefficients produced by bootstrapping a model based on 108 DINs. A relationship is considered long-term if still existing after 50 weeks.

Rule 1:

if coefficient $<=$ threshold

coefficient $=1 ;$ //relationship between market exists else

coefficient $=0$; // relationship between market does not exist end if

NZ50-AORD; exist $=0.63+0.35 ;$ not-exist $=0.37+0.65$

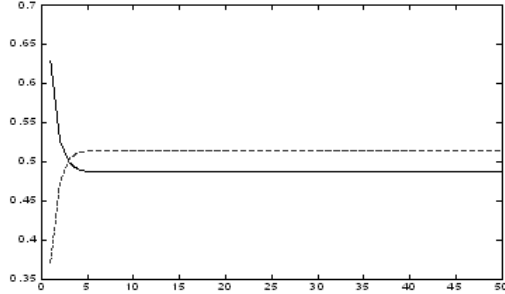

NZ50-HSLX; exist $=0.56+0.46$; not-exist $=0.44+0.54$

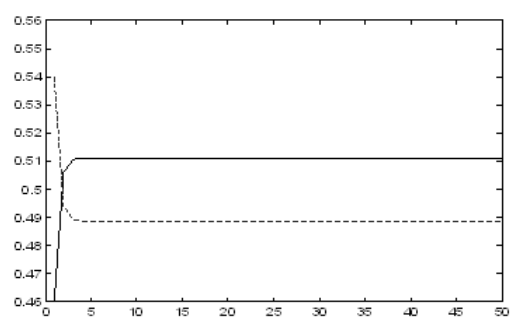

NZ50-KLSE; exist $=0.67+0.20$; not-exist $=0.33+0.80$

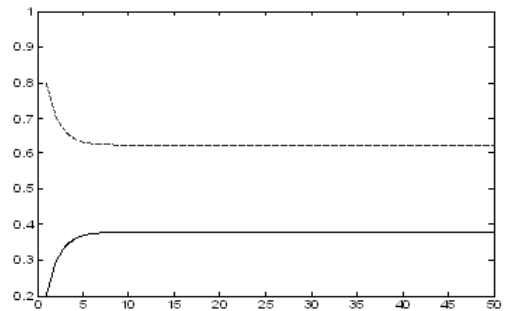

NZ50-N225; exist $=0.65+0.32$; not-exist $=0.35+0.68$

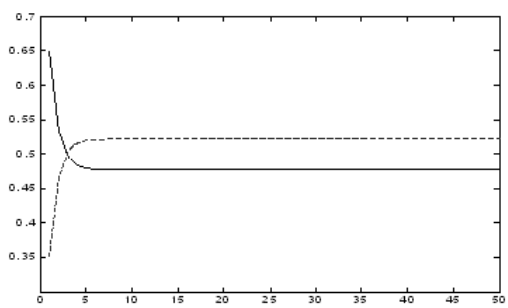

NZ50-STI; exist $=0.67+0.28$; not-exist $=0.33+0.72$

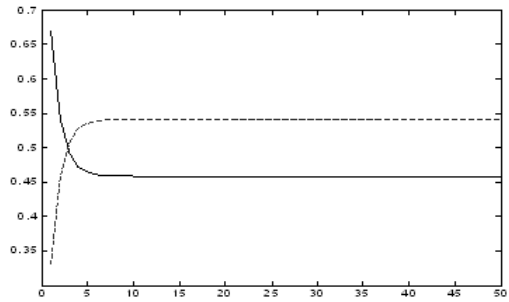

NZ50-NZ50; exist $=0.90+0.47 ;$ not-exist $=0.10+0.53$

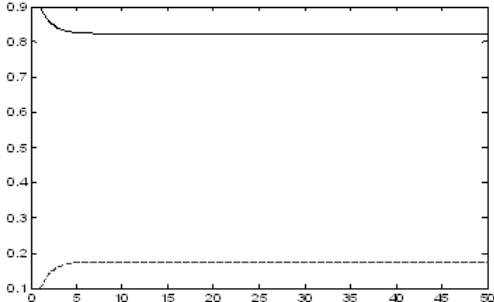

NZ50-JSX; exist $=0.66+0.46$; not-exist $=0.34+0.54$

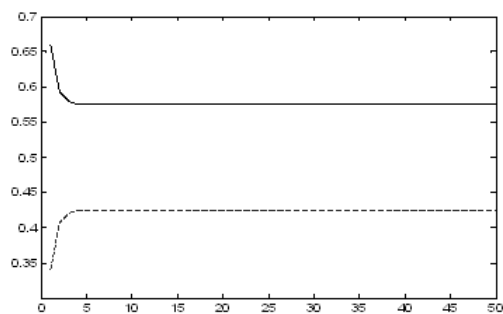

NZ50-KOSPI; exist $=0.71+0.27$; not-exist $=0.29+0.73$

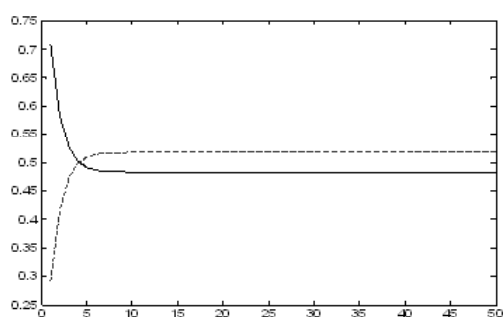

NZ50-SSE; exist $=0.66+0.42 ;$ not-exist $=0.34+0.58$

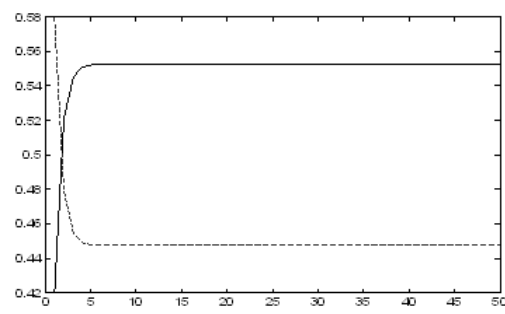

NZ50-TSEC; exist $=0.74+0.26$; not-exist $=0.26+0.74$

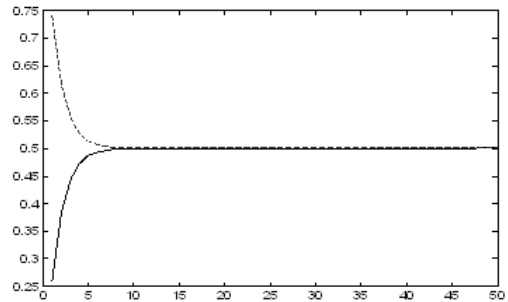

Figure 13: Markov chain model for NZ50 long-term relationships 
Figure 13 shows the types of relationships between markets. For instance, after a period of time, the lines in the NZ50-AORD graphics become stable or saturated at the level of 0.53 for "not exist" (dashed-line) and 0.47 for the "exist" (solid-line) concept. The graphics shows that the probability of NZ50 and AORD being in a short-term relationship is larger than its long-term relationship counterpart.

\section{Conclusion and Further Research}

Our study shows that extraction of dynamic interaction networks reveals important and complex interdependency among stock markets in Asia Pacific. We are able to capture relationships between multiple markets simultaneously, unlike most previous studies focusing on relationships between pairs of markets. The adaptive DIN model predicts stock index values with a reasonable degree of accuracy.

We would further like to work on a DIN extraction methodology that is not based on linear stochastic difference equation, allowing for the identification of non-linear relations among analysed markets. The next step is to introduce the common outside player, the US market, explicitly into the model. We would also like to extend the model to be able to capture global, local and personal behaviour of financial timeseries, and therefore improve prediction. The global model will be used to capture the trend in the whole problem space, the local model will extract local regression or specific behaviour from different sub-spaces of the whole problem space, and we are going to use the personalised model to cope with changes with current data or state of the system. Integrating the three models will provide a better framework for pattern recognition, adaptation and prediction for financial timeseries.

\section{Acknowledgements}

The software DRESt is based on GNetXP developed at KEDRI and accessible from http://www.aut.ac.nz/research/research_institutes/kedri/research_centres/centre_for_ bioinformatics/gene_network.htm.

We would like to thank Professor Dimitri Margaritis, Faculty of Business, Auckland University of Technology, for his comments and contribution.

\section{References}

[1] A. Antoniou, G. Pescetto, and A. Violaris, Modelling international price relationships and interdependencies between the stock index and stock index future markets of three EU countries: A Multivariate Analysis, Journal of Business Finance and Accounting 30: 645-667, 2003.

[2] R. Brown, Introduction to Random Signal Analysis and Kalman Filtering, Wiley, 1983. 
[3] G.M. Caporale, A. Serguieva, H. Wu, A mixed-game agent-based model for simulating financial contagion. In Proceedings of the 2008 Congress on Evolutionary Computation, 3420-3425, IEEE Press, 2008.

[4] Z. Chan, N. Kasabov, and L. Collins, A two-stage methodology for gene regulatory network extraction from time-course gene expression data, Expert System with Applications 30: 59-63, 2006.

[5] T.C. Chiang, S. Doong, Empirical analysis of stock returns and volatility: Evidence from seven Asian stock markets based on TAR-GARCH model, Review of Quantitative Finance and Accounting 17: 301-318, 2001.

[6] D. Collins, N. Biekpe, Contagion and interdependence in African stock markets, The South African Journal of Economics, 71(1): 181-194, 2003.

[7] P. D'haeseleer, S. Liang, and R. Somogyi, Gene expression data analysis and modelling. In Proceedings of the Pacific Symposium on Biocomputing, Hawaii, 1999.

[8] D. Goldberg, Genetic Algorithms in Search, Optimization and Machine Learning, Addison-Wesley, 1989.

[9] S. Goldfeld, R. Quandt, A Markov model for switching regressions, Journal of Econometrics 1(1): 3-16, 1973.

[10] J. Hamilton, Regime-switching models, Working Paper prepared for Palgrave Dictionary of Economics, University of California at San Diego, 2005.

[11] N. Kasabov, Evolving connectionist systems: Methods and applications in bioinformatics, Brain Study and Intelligent Machines, Springer, 2002.

[12] N. Kasabov, Z. Chan, V. Jain, I. Sidorov, D. Dimitrov, Gene regulatory network discovery from time-series gene expression data: A computational intelligence approach Presented at the: International Conference on Neural Information Processing, Calcutta, 2003. Published in Lecture notes in computer science 3316: 1344-1353, 2004.

[13] N. Kasabov, Global, local and personalised modelling and pattern discovery in bioinformatics: An integrated approach, Pattern Recognition Letters 28: 673$685,2007 a$.

[14] N. Kasabov, Evolving Connectionist Systems: The Knowledge Engineering Approach, Springer, 2007b.

[15] C. Kim, J. Piger, R. Startz, Estimation of Markov regime-switching regression models with endogenous switching, Research Division Working Papers 2003015C, Federal Reserve Bank of St. Louis, 2003 (revised 2005). 
[16] A. Masih, R. Masih, Dynamic modelling of stock market interdependencies: An empirical investigation of Australia and the Asian NICs, Working Papers 98-18: 1323-9244, University of Western Australia, 1998.

[17] G. Welch, G. Bishop, An Introduction to the Kalman Filter, Computer Science Working Papers TR95-041, University of North Carolina at Chapel Hill, 2006.

[18] A. Serguieva, T. Kalganova, T. Khan, An intelligent system for risk classification of stock investment projects, Journal of Applied Systems Studies 4 (2): 236-261, 2003.

[19] A. Serguieva, T. Khan: Knowledge representation in risk analysis, Business and Management Working Papers March'04: 1-21, Brunel University, 2004.

[20] A Serguieva, H. Wu, Computational intelligence in financial contagion analysis. Presented at the Seventh International Conference on Complex Systems, Boston, Massachusetts, 2007. Published in InterJournal on Complex Systems 2229: 1-12, 2008.

[21] Q. Song, N. Kasabov, Dynamic evolving neuro-fuzzy inference system (DENFIS): On-line learning and application for time-series prediction, IEEE Transactions of Fuzzy Systems 10: 144-154, 2002. 